\title{
Modulation of cellular bioenergetics by CO-releasing molecules and NO- donors inhibits the interaction of cancer cells with human lung microvascular endothelial cells
}

\author{
Marta Stojak $^{1}$, Patrycja Kaczara ${ }^{1}$, Roberto Motterlini ${ }^{2}$, Stefan Chlopicki ${ }^{1}$ \\ ${ }^{1}$ Jagiellonian Centre for Experimental Therapeutics (JCET), Jagiellonian University, Krakow, Poland \\ ${ }^{2}$ INSERM Unit 955, Equipe 12, Faculty of Medicine, University Paris-Est, Créteil, France
}

Correspondence to:

Stefan Chlopicki,

Bobrzynskiego, 14

30-348 Krakow, Poland

Fax: +48 122974615

Tel: +48126645464

E-mail: stefan.chlopicki@jcet.eu

\begin{abstract}
Interactions between cancer cells and the endothelium play a crucial role during metastasis. Here we examined the effects of a carbon monoxide-releasing molecule (CORM401) and a nitric oxide donor (PAPA NONOate) given alone or in combination on breast cancer cell adhesion and transmigration across the lung microvascular endothelium. We further explored whether the effects of $\mathrm{CO}$ and $\mathrm{NO}$ on cancer-endothelial cells interactions are linked with changes in cellular bioenergetics in breast cancer or endothelial cells. We found that CORM-401 and PAPA NONOate alone or in combination markedly decreased transmigration of breast cancer cells across human lung microvascular endothelial cells (hLMVEC), while cancer cell adhesion to the endothelium was diminished only by a combination of the two compounds. In hLMVEC, CORM-401 decreased glycolysis and stimulated mitochondrial respiration, while in breast cancer cells CORM-401 decreased both glycolysis and mitochondrial respiration. In contrast, PAPA NONOate decreased mitochondrial respiration and slightly stimulated glycolysis in both cell lines. When both donors were given together, mitochondrial respiration and glycolysis were both profoundly inhibited, and cancer-endothelial cells interactions were additively suppressed. Intercellular adhesion molecule-1 (ICAM-1), involved in breast cancer cell adhesion to hLMVECs, was downregulated by CORM-401 and PAPA NONOate, when applied alone, while a combination of both did not cause any enhancement of ICAM-1 downregulation.
\end{abstract}


Thus, our findings demonstrate that $\mathrm{CO}$ and NO differently affect cellular bioenergetics of cancer and endothelial cells and suggest that this phenomenon may contribute to additive antiadhesive and anti-transmigratory effects of $\mathrm{CO}$ and NO. We suggest that pharmacological attenuation of metabolism represents a novel paradigm in effective prevention of cancer cell interactions with the endothelium, that is an energy-demanding process.

\section{Keywords:}

CORM-401; PAPA NONOate; cellular bioenergetics; adhesion; transendothelial migration

\section{Introduction}

Breast cancer is the most common cancer affecting women worldwide and the most frequent cause of cancer death, with an annual incidence of approximately 1.4 million, and death rate of greater than 450,000 per year [1]. Tumor cell metastasis is responsible for the vast majority of cancer patient deaths [2,3]. Metastasis is a multistep process involving biochemical and physical interactions of cancer cells with the microenvironment that, due to its complexity, is far from being completely understood. During metastatic dissemination, cancer cells first detach from the primary tumor to permeate into the bloodstream and then extravasate and translocate to target organs, where they adapt to the new microenvironment and finally form metastases. Adhesion of metastatic cancer cells to the vascular endothelium and their subsequent transendothelial migration are critical steps in metastatic spread [4-7]. A number of endothelium-dependent mechanisms are responsible for favoring a pre-metastatic microenvironment, including a direct and indirect cross-talk between the two types of cells, which may promote the adhesion of cancerous cells to the endothelium. For example, factors released from dysfunctional endothelium activate NF- $\kappa \mathrm{B}$ and STAT3 signaling pathways within cancer cells and promote their invasiveness [8,9]. Still, very little is known about the mechanisms by which endothelial cells respond to the cancer cells during tumor progression and metastasis [9], as well as how the adhesiveness of cancer cells is affected by endothelial vasoprotective mediators including nitric oxide (NO) and carbon monoxide (CO).

Formation of a premetastatic niche appears to be associated with local endothelial dysfunction that precedes the development of systemic inflammation [10,11]. This involves impaired production of NO and increased expression of cell adhesion molecules (CAMs) $[12,13]$. One possibility is that cancer cells adhere to the endothelium by mechanisms similar to those used by leukocytes under inflammatory conditions, and that CAMs may regulate cancer-endothelial adhesion. Indeed, many tumors use also intercellular adhesion molecule 
(ICAM)-1, vascular cell adhesion molecule (VCAM)-1 and E-selectin expressed by endothelial cells to form adhesive bonds with the endothelium [9].

Endothelial NO, displaying anti-platelet and vasodilatory effects, inhibits adhesion of leukocytes to the endothelium [14,15]. NO also sensitizes tumor cells to chemotherapeutic compounds. In addition, various direct and indirect mechanisms have been proposed for the antitumor activities of NO [15,16]. Similarly, carbon monoxide (CO), another endotheliumderived molecule that serves as a signaling mediator eliciting potent anti-apoptotic, antiatherosclerotic, and anti-inflammatory effects [17-19], also modulates the polymorphonuclear leukocyte (PMN) adhesive phenotype and thus interferes with PMN recruitment to the affected organs [20,21]. Accordingly, endothelial NO and CO both inhibit the inflammatory and thrombotic mechanisms of the vascular wall [22]; thus their activity could regulate cancer cell adhesion to the endothelium in a manner similar to that seen in leukocyte trafficking. Given that cancer cell migration and metastasis are energy-demanding processes [23] and that both mediators, NO and CO, modulate cellular bioenergetics [24-28], in this study we explored whether a carbon monoxide-releasing molecule (CORM-401) and a nitric oxide donor (PAPA NONOate), given alone or in combination, modulate breast cancer cell adhesion and transmigration across the lung microvascular endothelium in association with any change in cellular metabolism, thus providing a mechanistic link for their anti-adhesive, anti-cancer properties.

\section{Material and methods}

\subsection{Reagents}

Reagents for cell culture, RPMI1640 GlutaMAX Medium, fetal bovine serum (FBS) were purchased from Gibco (Thermo Fisher Scientific). Microvascular Endothelial Cell Growth Medium was purchased from Cell Applications, Inc. Antibiotic Antimycotic Solution (AAS), 2-Deoxy-D-glucose (2-DG), (2E)-3-(3-Pyridinyl)-1-(4-pyridinyl)-2-propen-1-one (3 PO), carbonyl cyanide 4-(trifluoromethoxy)phenylhydrazone (FCCP), rotenone, antimycin A, glutamine, sodium pyruvate and trypsin were obtained from Sigma-Aldrich. Seahorse XF Base Medium Minimal DMEM was obtained from Agilent Technologies (USA). Oligomycin was obtained from Calbiochem. Glucose was obtained from Merck. Monoclonal anti-human CD54 (ICAM-1), anti-human CD106 (VCAM-1) and anti-human CD62E (E-selectin) were purchased from Ancell. Human interleukin-1 beta (IL-1 $\beta$ ) was obtained from Cell Signaling Technology. Calcein-AM, FITC Annexin V Apoptosis Detection Kit I, mouse anti-human 
CD54-PE, mouse anti-human CD106-PE-Cy5, mouse anti-human CD62E-APC antibody, PE

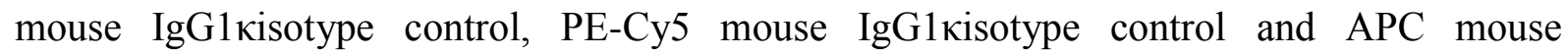
IgG1 kisotype control were purchased from BD Pharmingen. PAPA NONOate was purchased from Cayman. Stock solutions were prepared in $0.01 \mathrm{M} \mathrm{NaOH}$ and stored at $-80^{\circ} \mathrm{C}$. $0.01 \mathrm{M}$ $\mathrm{NaOH}$ was used as a vehicle in control experiments conducted with PAPA NONOate. Carbon monoxide-releasing molecule-401 (CORM-401), synthesized as described previously [29,30] was dissolved in PBS. Stock solutions were stored at $-80^{\circ} \mathrm{C}$ and protected from light in all experiments. PBS was used as a vehicle in control experiments conducted with CORM-401.

\subsection{Cell lines and cell culture}

Human lung microvascular endothelial cell line (hLMVEC) was purchased from the European Cell Culture Collection (Cell Applications, San Diego, CA, USA), human breast adenocarcinoma MDA-MB-231-luc2-tdTomato cell line stably expressing the firefly luciferase gene and tdTomato fluorescent protein was kindly provided by Prof. Joanna Wietrzyk (Ludwik Hirszfeld Institute of Immunology and Experimental Therapy, Polish Academy of Sciences). hLMVEC cells were maintained in Microvascular Endothelial Cell Growth Medium (Cell Applications), MDA-MB-231-luc2-tdTomato cells were cultured in RPMI1640 GlutaMAX Medium (Gibco) with 10\% FBS (Gibco), AAS (Sigma-Aldrich) containing 20 units of penicillin, $20 \mathrm{mg}$ streptomycin and $0.05 \mathrm{mg}$ amphotericin B. Cells were regularly tested for Mycoplasma contamination using the MycoAlert Mycoplasma DetectionKit (Lonza). Cells were cultured at $37^{\circ} \mathrm{C}$ in an atmosphere of $5 \% \mathrm{CO}_{2}$. In all experiments, cells between third and seventh passage were used.

\subsection{Flow cytometric phenotyping of hLMVECs}

hLMVEC cells were grown to confluence on 6-well tissue culture plates in fresh medium. Each well was stimulated differently with TNF- $\alpha(10 \mathrm{ng} / \mathrm{mL}), \mathrm{IL}-1 \beta(10 \mathrm{ng} / \mathrm{mL})$ for 3,6 and $24 \mathrm{~h}$. Cells were collected at the indicated time points using Accutase solution (SigmaAldrich). For each staining, expression of cell-surface ICAM-1 (intercellular adhesion molecule-1, CD54), E-selectin (CD62E) and VCAM-1 (vascular cell adhesion molecule-1, CD106) was measured by flow cytometry. hLMVECs were incubated with mouse anti-human CD54-PE (BD Pharmingen), mouse anti-human CD106-PE-Cy5 (BD Pharmingen) and mouse anti-human CD62E-APC antibody (BD Pharmingen), respectively. Background staining was performed by staining endothelial cells with PE mouse IgG1 isisotype control, PE-Cy5 mouse 


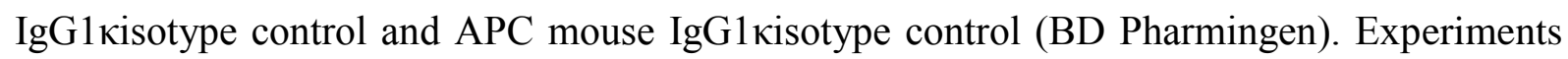
were conducted in triplicate and repeated three times. Flow cytometric analysis was performed on a BD LSR II (BD Biosciences) with 488 and $633 \mathrm{~nm}$ laser excitation. The BD FACSDiva software was employed for data acquisition and data analysis. Forward versus side scatter dot plots were used to gate on single intact cells. 10,000 cells were examined per one sample. PE and PE-Cy5 dyes were excited by a $488 \mathrm{~nm}$ laser and APC dye was excited by a $633 \mathrm{~nm}$ laser.

\subsection{Expression of ICAM-1}

hLMVECs were seeded into 96 -well plates $\left(3 \times 10^{4}\right.$ cells/well), in $100 \mu \mathrm{L}$ of medium per well and left to grow to confluence for $24-48 \mathrm{~h}$, and then incubated with IL-1 $\beta(10 \mathrm{ng} / \mathrm{mL})$ for $6 \mathrm{~h}$. After stimulation, endothelial cells were treated with CORM-401 and PAPA NONOate at various concentrations for $24 \mathrm{~h}(10,50,100 \mu \mathrm{M})$, or a combination of the NO-donor with CORM-401 $(50 \mu \mathrm{M})$ was performed as described above. Then, hLMVECs were incubated in the dark with mouse anti-human CD54-PE (BD Pharmingen) according to the manufacturer's protocol and stained with Hoechst 33342 (Life Technologies) for 10 minutes. The expression of surface ICAM-1 molecule was observed by fluorescence microscopy (ScanR screening system) in ten randomly selected visual fields for each well. The images were analyzed using Columbus 2.4.2 Software (Perkin Elmer). Experiments were performed in triplicate and repeated three times.

\subsection{Adhesion assay}

The hLMVECs were transferred into 96-well (seeding density $3 \times 10^{4}$ cells/well) plates and left to grow to confluence for $24-48 \mathrm{~h}$. Endothelial cells were stimulated with $10 \mathrm{ng} / \mathrm{mL} \mathrm{IL-1 \beta}$ for $6 \mathrm{~h}$. Prior to their use for the cell adhesion assay, MDA-MB-231-luc2-tdTomato were stimulated with $10 \mathrm{ng} / \mathrm{mL}$ IL-1 $\beta$ for 30 minutes and stained with Calcein-AM (BD Pharmingen) according to the manufacturer's instruction. Then, cancer cells were preincubated with various concentrations of CORM-401 and PAPA NONOate for 20 minutes. In combination experiments, cells were incubated with CORM-401 $(50 \mu \mathrm{M})$ in the presence of PAPA NONOate at various concentrations $(10,30,50 \mu \mathrm{M})$. Alternatively, cells were coincubated with $50 \mu \mathrm{M}$ PAPA NONOate in the presence of CORM-401 at different concentrations $(10,30,50 \mu \mathrm{M})$. Afterwards, labeled MDA-MB-231-luc2-tdTomato were left to adhere to the endothelial layer with $\mathrm{CO}-\mathrm{RM}$ and NO-donor, given alone or in combination, 
for 30 minutes at $37^{\circ} \mathrm{C}$. Non-adherent cells were gently washed twice with Dulbecco's phosphate-buffered saline (DPBS).

Adhesion blockade by specific monoclonal antibodies was performed to determine the contribution of ICAM-1, VCAM-1 and E-selectin to the MDA-MB-231-luc2-tdTomato cell adhesion to IL-1 $\beta$-treated hLMVECs. After stimulation of endothelial cells with IL-1 10 $\mathrm{ng} / \mathrm{mL}$ for $6 \mathrm{~h}$, monoclonal antibodies anti-ICAM-1, anti-VCAM-1 and anti-E-selectin (Ancell Immunology Research Products) at concentration $5 \mu \mathrm{g} / \mathrm{mL}$, were applied to the cells for 45 minutes. Then, cells were washed twice and labeled MDA-MB-231-luc2-tdTomato cells were added to the monolayer, and the assay performed as above.

In both assays, the attached cells were counted in ten randomly selected visual fields for each well. Pictures were taken using the ScanR screening system, containing a fully automated inverted fluorescence microscope (Olympus IX81), placed in an incubator with a system allowing for long-term microenvironment maintenance, appropriate for cell culturing (Life Imaging Services). Experiments were conducted in triplicate and repeated three times.

The images were analyzed using Columbus 2.4.2 Software (Perkin Elmer). The mean inhibition of adhesion for ten visual fields was calculated by using the equation: \% of control $=[$ the number of adhered cells in treated samples/the number of adhered cells in the control group $] \times 100 \%$.

\subsection{Transendothelial Migration Assay}

Cell migration was assayed in 24-well, 6.5-mm internal-diameter Transwell plates $(8.0 \mu \mathrm{m}$ pore size; BD Pharmingen). hLMVECs (seeding density $5 \times 10^{4}$ cells/insert) were first cultured for 24-48 $\mathrm{h}$ on the upper side of the filter. After confluent monolayer formation, hLMVECs were pretreated with $10 \mathrm{ng} / \mathrm{mL}$ IL-1 $\beta$ for $6 \mathrm{~h}$. Prior to use in transmigration assay, cancer cells were stimulated with $10 \mathrm{ng} / \mathrm{mL}$ IL-1 $\beta$ for 30 minutes. Then, MDA-MB-231-luc2-tdTomato cells (each $2.5 \times 10^{4}$ per well) were placed into upper chambers and CO-RM and NO-donor (5, $10,50 \mu \mathrm{M})$ were given alone or in combination, as described above. Furthermore, cells were co-incubated with oxidative phosphorylation inhibitor, oligomycin $(0.03 \mu \mathrm{g} / \mathrm{mL})$ and two glycolysis inhibitors, 2-Deoxy-D-glucose (2-DG, $50 \mathrm{mM}$ ) and (2E)-3-(3-Pyridinyl)-1-(4pyridinyl)-2-propen-1-one (3PO, $30 \mu \mathrm{M}$ ) alone or together. Lower chambers were filled with medium (20\% FBS) containing supernatants of tumor cell lines. After $24 \mathrm{~h}$ of co-culture, hLMVEC monolayers and non-migrating cancer cells on the upper surface of the membrane were removed by wiping with a cotton swab. Migrated cells on the undersides of the 
Transwell membranes were fixed with $0.5 \%$ glutaraldehyde in PBS for 10 minutes and stained with crystal violet solution according to the manufacturer's protocol. The number of cells on the lower face of the filter was counted in ten randomly selected visual fields under 200x magnification using light microscopy. Experiments were performed in triplicate and repeated three times.

\subsection{Annexin V-FITC/PI assay}

Dual staining of hLMVEC and MDA-MB-231-luc2-tdTomato cells with fluoresceinated annexin V (annexin V-FITC) and propidium iodide (PI) was performed using FITC Annexin V Apoptosis Detection Kit I (BD Pharmingen), according to the manufacturer's instruction. Briefly, cells were grown to confluence on 24-well plates. Then, cells were incubated with CORM-401 and PAPA NONOate alone or in combination for $24 \mathrm{~h}$. Moreover, both cell lines were treated with oligomycin, 2-DG and 3PO, alone or together for $24 \mathrm{~h}$. Treated and control cells were harvested by Accutase solution (Sigma-Aldrich), washed twice with cold PBS and resuspended in $100 \mu 1$ of binding buffer. Then, $5 \mu 1$ of annexin V-FITC and $5 \mu 1$ of PI staining solution were added to the cell suspension and cells were incubated in the dark for 15 minutes, at room temperature. Following the incubation, $400 \mu \mathrm{l}$ of binding buffer was added to each tube. Cell samples were placed on ice, away from light, and FITC and PI fluorescence were immediately measured using a BD LSR II flow cytometer. The frequency of viable cells, early apoptotic cells, late apoptotic and necrotic cells, were determined using BD FACSDiva software. The data were determined by three independent experiments.

\subsection{Analysis of Cellular Bioenergetics by Extracellular Flux Technology}

To measure mitochondrial function and glycolysis in adherent endothelial hLMVEC and cancer MDA-MB-231-luc2-tdTomato cells, a Seahorse Bioscience XFe 96 Analyzer was used. Cells were seeded into Seahorse XFe 96-well plates approximately $24 \mathrm{~h}$ before the experiment, according to the Seahorse protocol. For both cell lines, densities of 20,000 cells per well were selected in preliminary experiments as optimal. One day before the experiment, sensor cartridges were hydrated in XF calibrant and maintained at $37{ }^{\circ} \mathrm{C}$ in air without $\mathrm{CO}_{2}$. On the day of the experiment, cells were washed twice and incubated with bicarbonate-free low buffered assay medium for $1 \mathrm{~h}$ at $37{ }^{\circ} \mathrm{C}$ in air without $\mathrm{CO}_{2}$ prior to the beginning of the assay. The assay medium was freshly prepared on the day of the experiment by supplementation of XF Base Medium Minimal DMEM with metabolic substrates as in culture 
media used for propagation of these cell lines: glucose ( $1 \mathrm{~g} / \mathrm{L}$ for hLMVEC, $2 \mathrm{~g} / \mathrm{L}$ for MDAMB-231-luc2-tdTomato), glutamine (2 mM for both cell lines) and sodium pyruvate (1 mM for hLMVEC, 2 mM for MDA-MB-231-luc2-tdTomato); $\mathrm{pH}$ was adjusted to 7.4 with $\mathrm{NaOH}$. Changes in oxygen consumption rate (OCR) and extracellular acidification rate (ECAR) were assessed over time by sequential injections of vehicles, CORM-401 or PAPA NONOate in port $\mathrm{A}, 1 \mu \mathrm{g} / \mathrm{mL}$ oligomycin in port $\mathrm{B}, 0.8 \mu \mathrm{M}$ FCCP in port $\mathrm{C}$ and $0.5 \mu \mathrm{M} / 0.5 \mu \mathrm{M}$ rotenone/antymycin A in port D. Concentrations of oligomycin, FCCP, rotenone and antymycin A were optimized separately for both cell lines in preliminary experiments (data not shown). From the mitochondrial stress test, we determined key parameters of mitochondrial function by using a Seahorse XF Cell Mito Stress Test Report Generator for calculation of OCR values linked to: basal respiration, acute response, proton leak, maximal respiration, spare respiratory capacity, ATP production and non-mitochondrial respiration. Metabolic activity correlated with mitochondrial respiration or glycolysis was calculated as a sum of, respectively, OCR or ECAR values obtained during $1 \mathrm{~h}$ of measurement (ten time points) after injection of analyzed compounds from port A and before injection of oligomycin.

\subsection{Statistical analysis}

All results are presented as the mean value \pm standard deviation (SD). Statistical analysis was performed using GrapPad Prism 6 (San Diego, CA, USA). Statistical significance for the data was evaluated by one-way analysis of variance (ANOVA) followed by Tukey's significant differences multiple range test. $\mathrm{P}$ values $<0.05$ were considered statistically significant.

\section{Results}

\subsection{Involvement of ICAM-1 in the adhesion of cancer cells to the endothelium}

The potency of the proinflammatory cytokine IL-1 $\beta$ in comparison with TNF- $\alpha$ to stimulate ICAM-1, VCAM-1 and E-selectin expression in hLMVEC cells was determined by flow cytometry. In the non-stimulated hLMVECs, expression of ICAM-1 and VCAM-1 was present, but E-selectin was absent. Following stimulation of hLMVECs by IL-1 $\beta(10 \mathrm{ng} / \mathrm{mL})$ or TNF- $\alpha(10 \mathrm{ng} / \mathrm{mL})$ for 3, 6 and $24 \mathrm{~h}$, expression of ICAM-1 was substantially up-regulated (Fig. 1a), while the expression of E-selectin and VCAM-1 was only slightly increased (Fig. $1 \mathrm{~b}, \mathrm{c})$. As shown in Fig. 1a, the maximal induction of ICAM-1 was obtained $3 \mathrm{~h}$ after IL-1 $\beta$ or TNF- $\alpha$ stimulation of hLMVECs and persisted over time. The highest expression of VCAM-1 was observed 3-6 h after exposure of hLMVECs to IL-1 $\beta$ or TNF- $\alpha$ and slightly decreased 
thereafter (Fig. 1c). Similarly, the maximum level of E-selectin expression was detected $3 \mathrm{~h}$ after stimulation of endothelial cells by IL- $1 \beta$ and TNF- $\alpha$ but decreased over a longer period of time (Fig. 1b). In the functional adhesion assay, stimulation with IL-1 $\beta(10 \mathrm{ng} / \mathrm{mL})$ increased adhesion of breast cancer cells to endothelial cells to a greater extend as compared with TNF- $\alpha$ (10 ng/mL) (unpublished data). According to our results, IL-1 $\beta$ was chosen as a tool molecule to investigate the effect of CORM-401 and PAPA NONOate on the interaction between cancer and endothelial cells. In all experiments, hLMVECs were pretreated with IL$1 \beta(10 \mathrm{ng} / \mathrm{mL})$ for $6 \mathrm{~h}$ prior to co-incubation with fluorescence-labeled MDA-MB-231-luc2tdTomato cancer cells. To examine the adhesion molecules involved in the adhesion of MDAMB-231-luc2-tdTomato cancer cells to the endothelium, monoclonal blocking antibodies anti-ICAM-1, anti-VCAM-1 or anti-E-selectin, were used. Incubation of hLMVECs with antiICAM-1 antibody effectively decreased the adhesion of MDA-MB-231-luc2-tdTomato to the endothelial monolayer. As shown in Fig. 2, anti-ICAM-1 at a concentration $5 \mu \mathrm{g} / \mathrm{mL}$ inhibited breast cancer cell adhesion by $\sim 40 \%$, whereas the anti-VCAM-1 and anti-E-selectin antibodies did not reduce breast cancer cell adhesion (Fig. 2).

\subsection{Effect of CORM-401 and PAPA NONOate on ICAM-1 expression}

In order to verify whether CORM-401 and PAPA NONOate interfere with ICAM-1 expression, hLMVEC cells were pretreated with $10 \mathrm{ng} / \mathrm{mL}$ IL-1 $\beta$ for $6 \mathrm{~h}$, followed by treatment with CORM-401 and PAPA NONOate alone or combined for $24 \mathrm{~h}$. Expression of ICAM-1 was detected by fluorescence microscopy. Incubation with CORM-401 or PAPA NONOate alone caused a decrease in ICAM-1 expression (Fig. 3a, b, d). The combined treatment with CORM-401 and PAPA NONOate did not enhance the down-regulation of ICAM-1 induced by one of the compounds given alone (Fig. 3c, d).

\subsection{Effect of CORM-401 and PAPA NONOate on adhesion of cancer cells to the endothelium}

To explore the effect of CORM-401 and PAPA NONOate, given alone or in combination, on breast cancer cell adhesion to the endothelial monolayer, a functional adhesion assay was used, which was based on quantification of MDA-MB-231-luc2-tdTomato cells adhering to lung microvascular endothelium by fluorescence-labeled cell counts. Either CORM-401 or PAPA NONOate given alone, did not interfere with the adhesion of MDA-MB-231-luc2tdTomato to hLMVECs (Fig. 4a, b, d). However, combined treatment with CORM-401 and PAPA NONOate resulted in inhibition of breast cancer cell adhesion (Fig. 4c, d). 
3.4. Effect of CORM-401 and PAPA NONOate on transmigration of cancer cells across the endothelium

In order to assess the effect of CORM-401 and PAPA NONOate, given alone or in combination, on transmigration of cancer cells across lung microvascular endothelium, the Transwell assay was used. As shown in Fig. 4e, f, CORM-401 and PAPA NONOate given alone, significantly decreased transendothelial migration of MDA-MB-231-luc2-tdTomato cancer cells, in a dose-dependent manner. CORM-401 appeared to be more active in suppression of MDA-MB-231-luc2-tdTomato cell migratory activity $(54.1 \pm 15.6 \%$ for $5 \mu \mathrm{M}$; $46.9 \pm 15.5 \%$ for $10 \mu \mathrm{M} ; 25.8 \pm 6.7 \%$ for $50 \mu \mathrm{M}$ as compared to the control value), while PAPA NONOate was slightly less effective $(62.5 \pm 14.3 \%$ for $5 \mu \mathrm{M} ; 55.9 \pm 15.3 \%$ for $10 \mu \mathrm{M}$; $52.9 \pm 13.9 \%$ for $50 \mu \mathrm{M}$, of control value). We next examined the additive anti-transmigratory effect after combination of CORM-401 with PAPA NONOate. As shown in Fig. 4g, the application of CORM-401 $(50 \mu \mathrm{M})$ with PAPA NONOate $(10,50 \mu \mathrm{M})$, enhanced the inhibition of MDA-MB-231-luc2-tdTomato transmigration to $14.8 \pm 3.9 \%$ and $8.8 \pm 1.9 \%$, respectively. In comparison, the inhibition afforded by either compound alone was $25.8 \pm 6.7 \%$ for $50 \mu \mathrm{M}$ CORM-401 and 55.9 $\pm 15.3 \%(10 \mu \mathrm{M}), 52.9 \pm 13.9 \%(50 \mu \mathrm{M})$ for PAPA NONOate, respectively.

3.5. Effect of CORM-401 and PAPA NONOate on hLMVECs and MDA-MB-231-luc2tdTomato cell death induction

To exclude that the observed effects of CORM-401 and PAPA NONOate on cancer cell adhesion and transendothelial migration were related to cytotoxic activity of the tested compounds, an annexin V-FITC/PI assay was performed. The percentage of viable cells (annexin V-/PI-), early apoptotic (annexin V+/PI-), late apoptotic and necrotic (annexin $\mathrm{V}+\mathrm{PI}+$ ) cells upon treatment with CORM-401 and PAPA NONOate were determined (Fig. S1). As shown in Fig. S1, after CORM-401 and PAPA NONOate application alone (Fig. S1a, b, d, e) or in combination (Fig. S1c, f), the percentage of early apoptotic and also apoptotic and necrotic hLMVEC and MDA-MB-231-luc2-tdTomato cells were increased only after a very high concentration of CORM-401 and PAPA NONOate (500 $\mu \mathrm{M}$, for CORM-401, $>1 \mathrm{mM}$ for PAPA NONOate (data not shown), respectively). Therefore, the effects of CORM401 and PAPA NONOate given at concentrations below $100 \mu \mathrm{M}$ to investigate the effects of CO- and NO-donor on breast cancer cell adhesion to and transmigration across endothelial cells could not be related to cytotoxic and pro-apoptotic effects of these compounds. 


\subsection{Effect of CORM-401 and PAPA NONOate on mitochondrial respiration and glycolysis}

$\mathrm{CO}$ and NO are known to modulate mitochondrial respiration and glycolysis [25,27,31,32]. In order to analyze whether the effects of CORM-A1, CORM-401 and PAPA NONOate on the interactions between MDA-MB-231-luc2-tdTomato and hLMVECs cells are correlated with their effects on oxygen consumption rate (OCR) and extracellular acidification rate (ECAR), a Seahorse XF technique was used. In hLMVECs, CORM-401 induced a dose-dependent and transient increase in OCR accompanied by a stable decrease in ECAR (Fig. 5a, b), reflecting transiently increased mitochondrial respiration and profoundly decreased glycolysis. A mitochondrial stress test (MST) also revealed an increased proton leak (seen as higher OCR after oligomycin administration in CORM-401 pretreated cells as compared to the control, Fig. S2). PAPA NONOate induced a dose-dependent and transient decrease in OCR, accompanied by an increase in ECAR (Fig. 5c, d), but almost did not affect overall cellular bioenergetics encompassing both ATP-generating processes, analyzed as OCR and ECAR. When PAPA NONOate $(50 \mu \mathrm{M})$ was applied in combination with CORM-401 (50 $\mu \mathrm{M})$, then their effects on mitochondrial bioenergetics were comparable to those for CORM-401 given alone, whereas the effects on glycolytic bioenergetics clearly resulted from a combination of these compounds (PAPA NONOate given alone increased ECAR, CORM-401 given alone decreased ECAR, both compounds given in combination decreased ECAR, but to a lesser extent than CORM-401 given alone). Overall, mitochondrial and glycolytic cellular bioenergetics in hLMVEC cells were reduced by combined treatment with CORM-401 and PAPA NONOate (Fig. 5e, f). The effects of CORM-401 and PAPA NONOate on OCR and ECAR in hLMVEC non pre-incubated with IL-1 $\beta$ were comparable to cells pre-treated with L-1 $\beta$, and IL-1 $\beta$ alone did not modify either basal OCR or ECAR (Fig. 5g, h).

In MDA-MB-231-luc2-tdTomato cancer cells, all of the applied donors inhibited oxygen consumption in a dose-dependent manner but with different dynamics: CORM-401 induced a decrease in OCR, which was substantial and progressed over time (clearly seen also after FCCP application, Fig. 6a, b), reflecting decreased mitochondrial bioenergetics; PAPA NONOate induced a decrease in OCR, which was rapid and transient (Fig. 6c, d), relatively strongly decreasing mitochondrial bioenergetics. ECAR, which reflects glycolytic bioenergetics, was in MDA-MB-231-luc2-tdTomato cells, only mildly affected by PAPA NONOate, but more strongly by CORM-401. When PAPA NONOate $(50 \mu \mathrm{M})$ was applied in combination with CORM-401 $(50 \mu \mathrm{M})$, then the effect on mitochondrial bioenergetics was slightly lower than for the compounds given alone, whereas the effect on glycolytic 
bioenergetics was almost as strong as for CORM-401 given alone (Fig. 6e, f). Altogether, PAPA NONOate and CORM-401 in combination induced a stronger inhibitory effect on cellular bioenergetics in MDA-MB-231-luc2-tdTomato cancer cells when compared to each compound alone. IL-1 $\beta$ alone did not modified basal OCR and ECAR and the responses of MDA-MB-231-luc2-tdTomato cells to CORM-401 and PAPA NONOate in MDA-MB-231luc2-tdTomato cancer cells that were not stimulated by IL-1 $\beta$ were similar (Fig. 6g, h).

3.7. Effect of inhibitors of mitochondrial respiration and glycolysis on transmigration of cancer cells across the endothelium

In order to confirm whether the modulation of glycolysis and oxidative phosphorylation by CORM-401 $(50 \mu \mathrm{M})$ and PAPA NONOate $(50 \mu \mathrm{M})$ could indeed contribute to the inhibition of breast cancer cells, we assessed the effect of inhibitors of glycolysis and oxidative phosphorylation (Fig. 7a, b) on transendothelial migration. Oligomycin, 3PO and particularly 2 -DG given alone decreased transendothelial migration of breast cancer cells $(87.3 \pm 17.1 \%$ for oligomycin $0.03 \mu \mathrm{g} / \mathrm{mL}, 63.9 \pm 13.6 \%$ for $3 \mathrm{PO} 30 \mu \mathrm{M}, 45.4 \pm 11.6 \%$ for 2 -DG $50 \mathrm{mM}$ as compared to the control value). Moreover, combined application of 2-DG with oligomycin or 3PO with oligomycin did not enhance inhibition of MDA-MB-231-luc2-tdTomato transendothelial migration, compared with the values obtained after a single application of these compounds (Fig. 7c, d).

\subsection{Effect of inhibitors of mitochondrial respiration and glycolysis on cell death induction}

To explore whether the inhibition of metabolism was linked with cell death induction, the effects of the inhibitors of cellular bioenergetics on triggering apoptosis were assessed. The exposure of hLMVECs and MDA-MB-231-luc2-tdTomato cells to 2-DG, 3PO or oligomycin given alone significantly triggered apoptosis in both cell lines (Fig. S3a, b, c, d). The combination of 2-DG with oligomycin was more effective in apoptosis-induction than the combination of $3 \mathrm{PO}$ with 2-DG in both cell lines as compared with the percentage values found after a single application of the tested agents. Moreover, MDA-MB-231-luc2-tdTomato cells appeared to be more sensitive than hLMVEC cells to 2-DG, 3PO and oligomycin.

\section{Discussion}

The adhesion of cancer cells to the endothelium and their transendothelial migration represent a critical and an energy-demanding process in the metastatic cascade. In the present study we 
reported that both $\mathrm{CO}$ and NO delivered from a CO-RM (CORM-401) and a NO-donor (PAPA NONOate), respectively, prevented the adhesion of breast cancer cells and their transmigration across the lung microvascular endothelium, and exhibited additive effects in combined treatment. We report here that the additive effects of CORM-401 and PAPA NONOate on breast cancer cell interactions with the endothelium involve complementary attenuation of cellular bioenergetics. Despite the fact that ICAM-1 was involved in the adhesive interactions between breast cancer and endothelial cells, due to the fact that treatment with both CORM-401 and PAPA NONOate resulted in its downregulation, this still does not explain the additive effects of CORM-401 and PAPA NONOate. In turn, inhibitors of glycolysis and mitochondrial respiration efficiently inhibited transendothelial migration of cancer cells across the endothelium, supporting the notion that the cellular bioenergetics may be an important target for the anti-adhesive and anti-cancer actions of $\mathrm{CO}$ and NO.

In the present work, we used functional adhesion and transendothelial migration assays to study the effects of CORM-401 and PAPA NONOate on the interactions between breast cancer MDA-MB-231-luc2tdTomato cells and IL-1 $\beta$-activated hLMVECs. We demonstrated that the adhesion of breast cancer cells to hLMVECs was significantly inhibited after blocking ICAM-1, but not VCAM-1 nor E-selectin, which were also upregulated. These results suggest that ICAM-1 is an important player in breast cancer cell interactions with lung microvascular endothelial cells, consistent with other findings indicating that ICAM-1 is required for invasion of metastatic breast cancer cells [33]. However, it is worth mentioning that ICAM-1 is one of many various mechanisms involved in interactions between cancer and endothelial cells. Herein, we showed that CORM-401 and PAPA NONOate given alone down-regulated the expression of ICAM-1 on the hLMVECs' surface after IL-1 $\beta$ stimulation. Our results confirm that exogenous $\mathrm{CO}$ liberated from $\mathrm{CO}-\mathrm{RMs}$ and $\mathrm{NO}$ released from NO-donors inhibit the pro-inflammatory response of the endothelium [17,34,35]. The experiments using a combination of CO-RM with the NO-donor did not enhance the down-regulation of surface ICAM-1 expression in endothelial cells when compared with either compounds used alone. The lack of additive effects of $\mathrm{CO}$ and NO on ICAM-1 expression excludes the possibility that the complementary effects of $\mathrm{CO}$ and $\mathrm{NO}$, seen in adhesion and in particular transendothelial migration assays, depends on additive effects of CO and NO on ICAM-1 expression. This observation indicate that there must be something else as a target for $\mathrm{CO}$ and NO than only ICAM-1. At the same time this observation may suggest that both CO and NO target the same mechanism, which leads to down-regulation of ICAM-1 [36]. In fact, it was 
demonstrated that both CO [37] and NO [38] downregulate ICAM-1 through the inhibition of the NF- $\kappa \mathrm{B}$ signaling pathway. Although $\mathrm{CO}$ and NO may affect multiple pathways apart from the NF-кB, e.g. guanylyl cyclase, calcium-activated potassium channels, mitochondrial respiratory chain or p38 mitogen-activated protein kinases (p38 MAPK) [17,39], in the present work we focused on the effects of $\mathrm{CO}$ and $\mathrm{NO}$ on bioenergetics of cancer cells and the endothelium. We demonstrated that in endothelial cells, CORM-401 caused stable attenuation of glycolysis in contrast to transient stimulation of mitochondrial respiration, while PAPA NONOate decreased mitochondrial respiration with sustained stimulation of glycolysis. Treatment of endothelial cells with a combination of CORM-401 and PAPA NONOate efficiently inhibited both pathways of ATP-generation, leading to quiescence of cells. Similarly, in MDA-MB-231-luc2-tdTomato cancer cells, complementary effects of CORM401 and PAPA NONOate on metabolism were demonstrated: CORM-401 mildly decreased mitochondrial respiration with a pronounced effect on glycolysis while PAPA NONOate decreased mitochondrial metabolic activity and slightly increased glycolysis. The effects induced by PAPA NONOate on mitochondrial respiration were reversible, whereas those induced by CORM-401 on glycolysis were irreversible. When the donors of CO and NO were applied in combination (PAPA NONOate+CORM-401), mitochondrial respiration and glycolysis in cancer cells were both profoundly inhibited and cancer-endothelial cell interactions were additively inhibited. Based on these results, we suggest that inhibition of mitochondrial respiration and glycolysis in cancer cells accompanied by the inhibition of glycolysis linked to an anti-inflammatory effect in endothelial cells - afforded by combined treatment with donors of $\mathrm{CO}$ and NO (CORM-401, PAPA NONOate) - provide the mechanistic explanation for the additive effect of these compounds on cancer cell interactions with the endothelium.

It has been reported in cells of diverse origins that $\mathrm{CO}$ and $\mathrm{NO}$ affect cellular metabolism [24-28,39]. Wegiel et al. [24] showed that in cancer cells, CO modulates mitochondrial respiration and glycolysis, leading to metabolic exhaustion. Recently, we demonstrated in endothelial cells that CO liberated from CORM-401 activates mitochondrial respiration with a mild uncoupling effect while reducing glycolysis, thus shifting ATP production from glycolysis to oxidative phosphorylation [25,26]. Yamamoto et al. [28] demonstrated in cancer cells that $\mathrm{CO}$, by the inhibition of cystathionine $\beta$-synthase (CBS), modulates activity of phosphofructokinase/fructose bisphosphatase type-3 (PFKFB3) and shunts glucose from glycolysis towards the pentose phosphate pathway, which can be important in anti-oxidative 
defense. Dranka et al. [27] showed in endothelial cells that NO released from DETA NONOate reversibly decreases mitochondrial respiration. Both $\mathrm{CO}$ and $\mathrm{NO}$ are capable of inhibiting mitochondrial cytochrome oxidase, but their physiological importance strongly depends on kinetics of release and local oxygen conditions [31]. Here we observed in our models that the effects on mitochondrial bioenergetics were dominated by a molecule which was delivered more quickly and in higher amount - CO from CORM-401 over NO from PAPA NONOate. Despite a number of reports demonstrating the effects of $\mathrm{CO}$ and NO on cellular metabolism, we show here for the first time that the anti-adhesive and antitransmigratory effects of $\mathrm{CO}$ and $\mathrm{NO}$ on cancer-endothelium interactions may be mediated by their effects on cellular bioenergetics. Lung microvascular endothelial cells, treated by combined CORM-401 and PAPA NONOate, displayed moderately decreased bioenergetics that could explain the anti-adhesive phenotype of endothelial cells. Bioenergetic activity of breast cancer cells was decreased more substantially, resulting in reduced transmigratory potential. Cancer cells are usually considered as mainly glycolytic for ATP production; however, it is important to stress that cancer cells also contain functional mitochondria and mitochondrial metabolism is not only an alternative source for production of ATP, but it is required for cancer growth $[40,41]$. Transmigration of cancer cells across the endothelium is an energy-demanding process, both from the side of the cancer cell as well as the endothelium. An invasive cancer cell, in order to reorganize and reassemble its cytoskeleton as well as to produce proteases to penetrate across the endothelium, needs ATP, which is generated mainly in mitochondria [42-44]. On the other side, an activated endothelial cell needs ATP derived from glycolysis for the activation of a pro-inflammatory response and the interaction with cancer cells [45]. Indeed, it was shown that inhibition of glycolysis at the level of PFKFB3 decreased the expression of adhesive molecules in endothelial cells, resulting in reduced adhesion and transmigration of cancer cells across the endothelium [46].

To support the notion that the inhibition of cellular bioenergetics results in the suppression of cancer-endothelial cell interactions, we demonstrated that 2-DG and 3PO, inhibitors of hexokinase 2 and 6-phosphofructo-2-kinase/fructose-2,6-biphosphatase 3 (PFKFB3), respectively, and oligomycin, an inhibitor of ATP synthase, diminished transendothelial migration of breast cancer cells. However, all inhibitors showed a clear proapoptotic effect, and their cytotoxicity towards cancer cells seems to be potentiated by the combination of inhibition of glycolysis and mitochondrial respiration. Interestingly, the effect of $\mathrm{CO}$ and $\mathrm{NO}$ on cellular metabolism was comparable to effect exerted by inhibitors of 
glycolysis or oligomycin, but the anti-transmigratory effect was strongly additive without evident cytotoxicy of the single and combined treatment. The results clearly show that suppression of cellular metabolism results not only in the inhibition of transendothelial migration of cancer cells, but also triggers apoptosis. Nevertheless, results with the inhibitors of metabolism confirm our hypothesis that the inhibition of cellular bioenergetics can inhibit transendothelial migration of cancer cells. On the other hand, these results also suggest that the inhibition of metabolism can trigger cell death not only in cancer cells but also host cells. Thus, it is tempting to speculate that it is better to design the strategy to inhibit cancerendothelium interactions based on endogenous mediators such as $\mathrm{NO}$ or $\mathrm{CO}$, which mildly and/or transiently modulate metabolism, rather than on potent inhibitors of oxidative phosphorylation and glycolysis.

\section{Conclusions}

Recently, cellular metabolism has been emerging as a potent target for cancer therapies, because it supports cancer progression and metastasis by delivering multiple metabolites and metabolic by-products [47-49]. Understanding the similarities and differences in metabolism between endothelial cells and cancer cells, and the role of CO and NO in metabolic regulation of different cell types, implies that cellular metabolism could be an attractive target for antitumor strategies. In view of the data presented here, we suggest that complementary effects of $\mathrm{CO}$ and $\mathrm{NO}$ on cancer cell metabolism may contribute to additive anti-adhesive and anti-transmigratory effects, particularly clearly seen in transendothelial migration assays. Accordingly, our data point out that pharmacological attenuation of metabolism of cancer cells and the endothelium by $\mathrm{CO}$ - and NO-donors, and possibly other molecules, represent an attractive target for preventing the interaction between cancer and endothelial cells.

\section{Acknowledgments}

Project financed by Polish National Science Centre, decision no DEC-2013/08/M/NZ7/01034, and by The National Centre for Research and Development, no STRATEGMED1/233226/11/NCBR/2015. We thank Prof. Joanna Wietrzyk (Hirszfeld Institute of Immunology and Experimental Therapy, Polish Academy of Sciences) for 
providing the MDA-MB-231-luc2-tdTomato breast cancer cells in the frame of the collaborative STRATEGMED1/233226/11/NCBR/2015 project.

Declarations of interest:

None

\section{Author contributions}

Conceived and designed research: MS and SC. Performed the experiments: MS and PK. Analysed the data: MS and PK. Interpretation of results: MS, PK, RM and SC. Prepared figures: MS and PK. Drafted manuscript: MS and SC. Edited and revised manuscript: PK and RM. Preparation of final manuscript - MS and SC. All authors have corrected or have approved the final version of the manuscript.

\section{References:}

[1] R. Burton, R. Bell, The global challenge of reducing breast cancer mortality., Oncologist. 18 (2013) 1200-2. doi:10.1634/theoncologist.2013-0315.

[2] S.A. Patel, M.A. Dave, R.G. Murthy, K.Y. Helmy, P. Rameshwar, Metastatic breast cancer cells in the bone marrow microenvironment: novel insights into oncoprotection, Oncol. Rev. 5 (2011) 1-10. doi:10.1007/s12156-010-0071-y.Metastatic.

[3] A.J. Redig, S.S. McAllister, Breast cancer as a systemic disease: a view of metastasis., J. Intern. Med. 274 (2013) 113-26. doi:10.1111/joim.12084.

[4] C.L. Chaffer, R.A. Weinberg, A Perspective on Cancer Cell Metastasis, Science (80-. ). 331 (2011) 1559-1564. doi:10.1126/science.1203543.

[5] V. Plaks, C.D. Koopman, Z. Werb, Z.W. Vicki Plaks, Charlotte D. Koopman, V. Plaks, C.D. Koopman, Z. Werb, Circulating Tumor Cells, Science. 341 (2013) 1186-8. doi:10.1126/science.1235226.

[6] D. Miriam, U. Schumacher, S.A. Brooks, Metastasis Research Protocols, Second Edi, 1969. doi:10.1007/978-1-4939-2842-2.

[7] A.S. Azevedo, G. Follain, S. Patthabhiraman, S. Harlepp, J.G. Goetz, Metastasis of circulating tumor cells: Favorable soil or suitable biomechanics, or both?, Cell Adhes. Migr. 9 (2015) 345-356. doi:10.1080/19336918.2015.1059563.

[8] J.W. Franses, N.C. Drosu, W.J. Gibson, V.C. Chitalia, E.R. Edelman, Dysfunctional endothelial cells directly stimulate cancer inflammation and metastasis, Int. J. Cancer. 133 (2013) 1334-1344. doi:10.1002/ijc.28146.

[9] K.-J. Kim, S. Kwon, J. Yun, H. Jeong, H. Kim, E.H. Lee, S. Ye, C. Cho, STAT3 activation in endothelial cells is important for tumor metastasis via increased cell adhesion molecule expression, Oncogene. (2017) 1-15. doi:10.1038/onc.2017.148. 
[10] H. Peinado, S. Rafii, D. Lyden, Inflammation Joins the "Niche," Cancer Cell. 14 (2008) 347-349. doi:10.1016/j.ccr.2008.10.012.

[11] S.I. Grivennikov, F.R. Greten, M. Karin, Immunity, inflammation, and cancer, Cell. 140 (2010) 883-899. doi:10.1016/j.cell.2010.01.025 [doi].

[12] C.T. Mierke, C.T. Mierke, Role of the Endothelium during Tumor Cell Metastasis: Is the Endothelium a Barrier or a Promoter for Cell Invasion and Metastasis?, J. Biophys. 2008 (2008) 1-13. doi:10.1155/2008/183516.

[13] L. Zhang, M. Zeng, B.M. Fu, Inhibition of endothelial nitric oxide synthase decreases breast cancer cell MDA-MB-231 adhesion to intact microvessels under physiological flows, Am J Physiol Hear. Circ Physiol. 310 (2016) H1735-47. doi:10.1152/ajpheart.00109.2016.

[14] J. Loscalzo, Nitric oxide insufficiency, platelet activation, and arterial thrombosis., Circ. Res. 88 (2001) 756-762. doi:10.1161/hh0801.089861.

[15] Y. Lu, T. Yu, H. Liang, J. Wang, J. Xie, J. Shao, Y. Gao, S. Yu, S. Chen, L. Wang, L. Jia, Nitric oxide inhibits hetero-adhesion of cancer cells to endothelial cells: restraining circulating tumor cells from initiating metastatic cascade., Sci. Rep. 4 (2014) 4344. doi:10.1038/srep04344.

[16] W. Xu, L.Z. Liu, M. Loizidou, M. Ahmed, I.G. Charles, The role of nitric oxide in cancer., Cell Res. 12 (2002) 311-320. doi:10.1038/sj.cr.7290133.

[17] L.E. Otterbein, F.H. Bach, J. Alam, M. Soares, H. Tao Lu, M. Wysk, R.J. Davis, R.A. Flavell, A.M. Choi, Carbon monoxide has anti-inflammatory effects involving the mitogen-activated protein kinase pathway., Nat. Med. 6 (2000) 422-428. doi:10.1038/74680.

[18] R. Motterlini, L.E. Otterbein, The therapeutic potential of carbon monoxide, Nat. Rev. Drug Discov. 9 (2010) 728-743. doi:10.1038/nrd3228.

[19] R. Motterlini, P. Sawle, J. Hammad, B.E. Mann, T.R. Johnson, C.J. Green, R. Foresti, Vasorelaxing effects and inhibition of nitric oxide in macrophages by new ironcontaining carbon monoxide-releasing molecules (CO-RMs), Pharmacol. Res. 68 (2013) 108-117. doi:10.1016/j.phrs.2012.12.001.

[20] P. Urquhart, G. Rosignoli, D. Cooper, R. Motterlini, M. Perretti, Carbon monoxidereleasing molecules modulate leukocyte-endothelial interactions under flow., J. Pharmacol. Exp. Ther. 321 (2007) 656-662. doi:10.1124/jpet.106.117218.

[21] K. Inoue, E.K. Patterson, A. Capretta, A.R. Lawendy, D.D. Fraser, G. Cepinskas, Carbon Monoxide-Releasing Molecule-401 Suppresses Polymorphonuclear Leukocyte Migratory Potential by Modulating F-Actin Dynamics, Am. J. Pathol. 187 (2017) 1121-1133. doi:10.1016/j.ajpath.2016.12.025.

[22] R.F. Furchgott, D. Jothianandan, Endothelium-dependent and -independent vasodilation involving cyclic GMP: relaxation induced by nitric oxide, carbon monoxide and light., Blood Vessels. 28 (1991) 52-61. http://www.ncbi.nlm.nih.gov/pubmed/1848126 (accessed December 19, 2017).

[23] T. Han, D. Kang, D. Ji, X. Wang, W. Zhan, M. Fu, H.B. Xin, J. Bin Wang, How does cancer cell metabolism affect tumor migration and invasion?, Cell Adhes. Migr. 7 
(2013) 395-403. doi:10.4161/cam.26345.

[24] B. Wegiel, D. Gallo, E. Csizmadia, C. Harris, J. Belcher, G.M. Vercellotti, N. Penacho, P. Seth, V. Sukhatme, A. Ahmed, P.P. Pandolfi, L. Helczynski, A. Bjartell, J.L. Persson, L.E. Otterbein, Carbon monoxide expedites metabolic exhaustion to inhibit tumor growth, Cancer Res. 73 (2013) 7009-7021. doi:10.1158/0008-5472.CAN-131075.

[25] P. Kaczara, R. Motterlini, G.M. Rosen, B. Augustynek, P. Bednarczyk, A. Szewczyk, R. Foresti, S. Chlopicki, Carbon monoxide released by CORM-401 uncouples mitochondrial respiration and inhibits glycolysis in endothelial cells: A role for mitoBK $<$ inf $>\mathrm{Ca}</ \mathrm{inf}>$ channels, Biochim. Biophys. Acta - Bioenerg. 1847 (2015) 1297-1309. doi:10.1016/j.bbabio.2015.07.004.

[26] P. Kaczara, R. Motterlini, K. Kus, A. Zakrzewska, A.Y. Abramov, S. Chlopicki, Carbon monoxide shifts energetic metabolism from glycolysis to oxidative phosphorylation in endothelial cells, FEBS Lett. 590 (2016) 3469-3480. doi:10.1002/1873-3468.12434.

[27] B.P. Dranka, B.G. Hill, V.M. Darley-Usmar, Mitochondrial reserve capacity in endothelial cells: The impact of nitric oxide and reactive oxygen species, Free Radic. Biol. Med. 48 (2010) 905-914. doi:10.1016/j.freeradbiomed.2010.01.015.

[28] T. Yamamoto, N. Takano, K. Ishiwata, M. Ohmura, Y. Nagahata, T. Matsuura, A. Kamata, K. Sakamoto, T. Nakanishi, A. Kubo, T. Hishiki, M. Suematsu, Reduced methylation of PFKFB3 in cancer cells shunts glucose towards the pentose phosphate pathway, Nat. Commun. 5 (2014) 1-16. doi:10.1038/ncomms4480.

[29] S.H. Crook, B.E. Mann, A.J.H.M. Meijer, H. Adams, P. Sawle, D. Scapens, R. Motterlini, $[\mathrm{Mn}(\mathrm{CO}) 4\{\mathrm{~S} 2 \mathrm{CNMe}(\mathrm{CH} 2 \mathrm{CO} 2 \mathrm{H})\}]$, a new water-soluble CO-releasing molecule, Dalt. Trans. 40 (2011) 4230. doi:10.1039/c1dt10125k.

[30] S. Fayad-Kobeissi, J. Ratovonantenaina, H. Dabiré, J.L. Wilson, A.M. Rodriguez, A. Berdeaux, J.L. Dubois-Randé, B.E. Mann, R. Motterlini, R. Foresti, Vascular and angiogenic activities of CORM-401, an oxidant-sensitive CO-releasing molecule, Biochem. Pharmacol. 102 (2016) 64-77. doi:10.1016/j.bcp.2015.12.014.

[31] C.E. Cooper, G.C. Brown, The inhibition of mitochondrial cytochrome oxidase by the gases carbon monoxide, nitric oxide, hydrogen cyanide and hydrogen sulfide: chemical mechanism and physiological significance, J. Bioenerg. Biomembr. 40 (2008) 533539. doi:10.1007/s10863-008-9166-6 [doi].

[32] J.L. Wilson, F. Bouillaud, A.S. Almeida, H.L. Vieira, M.O. Ouidja, J.L. Dubois-Randé, R. Foresti, R. Motterlini, Carbon monoxide reverses the metabolic adaptation of microglia cells to an inflammatory stimulus, Free Radic. Biol. Med. 104 (2017) 311323. doi:10.1016/j.freeradbiomed.2017.01.022.

[33] C. Rosette, R.B. Roth, P. Oeth, A. Braun, S. Kammerer, J. Ekblom, M.F. Denissenko, Role of ICAM1 in invasion of human breast cancer cells, Carcinogenesis. 26 (2005) 943-950. doi:10.1093/carcin/bgi070.

[34] J.L. Wallace, Nitric oxide as a regulator of inflammatory processes, Mem. Inst. Oswaldo Cruz. 100 (2005) 5-9. doi:10.1590/S0074-02762005000900002.

[35] C. Bergstraesser, S. Hoeger, H. Song, L. Ermantraut, M. Hottenrot, T. Czymai, M. 
Schmidt, M. Goebeler, N. Ponelies, C. Stich, R. Loesel, G. Molema, M. Seelen, W. Van Son, B.A. Yard, N. Rafat, Inhibition of VCAM-1 expression in endothelial cells by CORM-3: The role of the ubiquitin-proteasome system, p38, and mitochondrial respiration, Free Radic. Biol. Med. 52 (2012) 794-802.

doi:10.1016/j.freeradbiomed.2011.11.035.

[36] S. Kempe, H. Kestler, A. Lasar, T. Wirth, NF- $\kappa$ B controls the global pro-inflammatory response in endothelial cells: Evidence for the regulation of a pro-atherogenic program, Nucleic Acids Res. 33 (2005) 5308-5319. doi:10.1093/nar/gki836.

[37] B. Sun, X. Zou, Y. Chen, P. Zhang, G. Shi, Preconditioning of carbon monoxide releasing molecule-derived CO attenuates LPS-induced activation of HUVEC, Int. J. Biol. Sci. 4 (2008) 270-278. doi:10.7150/ijbs.4.270.

[38] B. V Khan, D.G. Harrison, M.T. Olbrych, R.W. Alexander, R.M. Medford, Nitric oxide regulates vascular cell adhesion molecule 1 gene expression and redox-sensitive transcriptional events in human vascular endothelial cells, Immunology. 93 (1996) 9114-9119. doi:10.1073/pnas.93.17.9114.

[39] C. Szabo, Gasotransmitters in cancer: from pathophysiology to experimental therapy, Nat. Rev. Drug Discov. 15 (2015) 185-203. doi:10.1038/nrd.2015.1.

[40] S.E. Weinberg, N.S. Chandel, Targeting mitochondria metabolism for cancer therapy, Nat. Chem. Biol. 11 (2014) 9-15. doi:10.1038/nchembio.1712.

[41] C.Y. Tang, C. Mauro, Similarities in the metabolic reprogramming of immune system and endothelium, Front. Immunol. 8 (2017). doi:10.3389/fimmu.2017.00837.

[42] V.S. LeBleu, J.T. O’Connell, K.N. Gonzalez Herrera, H. Wikman, K. Pantel, M.C. Haigis, F.M. de Carvalho, A. Damascena, L.T. Domingos Chinen, R.M. Rocha, J.M. Asara, R. Kalluri, PGC-1 $\alpha$ mediates mitochondrial biogenesis and oxidative phosphorylation in cancer cells to promote metastasis, Nat. Cell Biol. 16 (2014) 9921003. doi:10.1038/ncb3039.

[43] J. Zhao, J. Zhang, M. Yu, Y. Xie, Y. Huang, D.W. Wolff, P.W. Abel, Y. Tu, Mitochondrial dynamics regulates migration and invasion of breast cancer cells HHS Public Access, Oncogene. 32494 (2013) 4814-4824. doi:10.1038/onc.2012.494.

[44] M. Fujita, K. Imadome, T. Imai, Metabolic characterization of invaded cells of the pancreatic cancer cell line, PANC-1, Cancer Sci. 108 (2017) 961-971. doi:10.1111/cas.13220.

[45] R. Foresti, S.K. Bains, T.S. Pitchumony, L.E. De Castro Brás, F. Drago, J.L. DuboisRandé, C. Bucolo, R. Motterlini, Small molecule activators of the Nrf2-HO-1 antioxidant axis modulate heme metabolism and inflammation in BV2 microglia cells, Pharmacol. Res. 76 (2013) 132-148. doi:10.1016/j.phrs.2013.07.010.

[46] A.R. Cantelmo, L.C. Conradi, A. Brajic, J. Goveia, J. Kalucka, A. Pircher, P. Chaturvedi, J. Hol, B. Thienpont, L.A. Teuwen, S. Schoors, B. Boeckx, J. Vriens, A. Kuchnio, K. Veys, B. Cruys, L. Finotto, L. Treps, T.E. Stav-Noraas, F. Bifari, P. Stapor, I. Decimo, K. Kampen, K. De Bock, G. Haraldsen, L. Schoonjans, T. Rabelink, G. Eelen, B. Ghesquière, J. Rehman, D. Lambrechts, A.B. Malik, M. Dewerchin, P. Carmeliet, Inhibition of the Glycolytic Activator PFKFB3 in Endothelium Induces Tumor Vessel Normalization, Impairs Metastasis, and Improves Chemotherapy, Cancer 
Cell. 30 (2016) 968-985. doi:10.1016/j.ccell.2016.10.006.

[47] P.E. Porporato, S. Dhup, R.K. Dadhich, T. Copetti, P. Sonveaux, Anticancer targets in the glycolytic metabolism of tumors: A comprehensive review, Front. Pharmacol. AUG (2011) 1-18. doi:10.3389/fphar.2011.00049.

[48] L. Galluzzi, O. Kepp, M.G. Vander Heiden, G. Kroemer, Metabolic targets for cancer therapy, Nat. Rev. Drug Discov. 12 (2013) 829-846. doi:10.1038/nrd4145.

[49] D. Verdegem, S. Moens, P. Stapor, P. Carmeliet, Endothelial cell metabolism: parallels and divergences with cancer cell metabolism, Cancer Metab. 2 (2014) 19. doi:10.1186/2049-3002-2-19. 